\title{
End-Stage Renal Disease Attributed to Acute Tubular Necrosis in the United States, 2001-2010
}

\author{
Robert N. Foley ${ }^{a, b}$ Donal J. Sexton ${ }^{a}$ Scott Reule ${ }^{a, b}$ Craig Solid $^{a}$ \\ Shu-Cheng Chen ${ }^{a}$ Allan J. Collins ${ }^{a, b}$ \\ ${ }^{a}$ Chronic Disease Research Group, Minneapolis Medical Research Foundation and ${ }^{b}$ Department of Medicine, \\ University of Minnesota, Minneapolis, Minn., USA
}

\section{Key Words}

Acute tubular necrosis $\cdot$ Incidence $\cdot$ Dialysis outcomes

\begin{abstract}
Background/Aims: Though end-stage renal disease (ESRD) is increasingly attributed to acute tubular necrosis (ATN), contemporary trends in the rates of incidence and recovery of renal function are poorly defined. Hence, we set out to describe the clinical epidemiology of ESRD due to ATN between 2001 and 2010. Methods: We examined United States Renal Data System data $(n=1,070,490)$ for 2001 through 2010 to calculate the incidence rates and rates of renal recovery and death for patients with ESRD due to ATN treated with renal replacement therapy (RRT, $n=27,603)$. Results: Standardized incidence ratios increased between 2001-2002 and 2009-2010 in the overall population (ratio 2.14), having risen in all demographic subgroups examined. Recovery of renal function was more likely in patients with ATN than in matched controls (cumulative incidence $23 \%$ vs. $2 \%$ at 12 weeks, $34 \%$ vs. $4 \%$ at 1 year), as was death (cumulative incidence $38 \%$ vs. $27 \%$ at 1 year). Hazards ratios for renal recovery increased stepwise with year of RRT inception to 1.34 (95\% confidence interval 1.24-1.45) for 2009-2010 (vs. 2001-2002). In contrast, hazards ratios for death declined stepwise to $0.83(0.79-0.87)$ in 2009-2010. Conclusion: While the incidence of ESRD attributed to ATN has increased,
\end{abstract}

prospects of renal recovery and survival have also increased. Despite substantial mortality risk on RRT, renal recovery is not a rare occurrence.

(c) 2015 S. Karger AG, Basel

\section{Introduction}

Acute tubular necrosis (ATN) is a frequent occurrence in patients admitted to hospital, especially patients whose illnesses necessitate admission to intensive care units. ATN and other causes of acute kidney injury (AKI) have long been associated with undesirable short-term outcomes, such as longer hospital stays, higher costs, and heightened mortality, and it has become apparent that they also foreshadow progressive chronic kidney disease, end-stage renal disease (ESRD), and long-term mortality [1-7]. Surprisingly, as few if any contemporary clinical epidemiological studies of ESRD due to ATN are available, the growth of ESRD from ATN is not well characterized. Of potential importance for clinical decision making, little is known regarding the outcomes on mainte-

This study was performed as a deliverable under Contract No. HHSN267200715002C (National Institute of Diabetes and Digestive and Kidney Diseases, National Institutes of Health, Bethesda, Md., USA). The authors have no conflicts of interest with its subject matter.

\section{KARGER 125}

C 2015 S. Karger AG, Basel

0250-8095/15/0411-0001\$39.50/0

E-Mail karger@karger.com

www.karger.com/ajn
Robert N. Foley, MB

Chronic Disease Research Group, Minneapolis Medical Research Foundation

914 South 8th Street, Suite S-406

Minneapolis, MN 55404 (USA)

E-Mail rfoley@cdrg.org 
nance renal replacement therapy (RRT). Hence, we set out to describe the clinical epidemiology of ESRD due to ATN between 2001 and 2010.

\section{Methods}

Objectives

The principal objectives of this study were to evaluate the trends in standardized incidence ratios, relative to rates in 20012002, of ESRD due to ATN, necessitating RRT in the United States up to 2010. For clinical outcomes after the initiation of RRT, we set out to compare the rates of renal recovery and death in matched patients with and without ATN, and to calculate the hazards ratios for these outcomes among patients with ATN.

\section{Subjects}

In this retrospective study, we used United States Renal Data System (USRDS) standard analysis files to study US patients who initiated maintenance RRT between 2001 and $2010(n=1,070,490)$. Baseline characteristics at initiation of RRT were determined from the Centers for Medicare and Medicaid (CMS) Medical Evidence Report (form CMS-2728). By federal requirement, this form had to be submitted for all new patients starting RRT in the United States and resultant data are housed in the USRDS Medevid95 and Medevid05 files. The Medical Evidence Report form has changed twice in the past two decades, in 1995 and 2005. Unlike previous iterations, the 2005 form includes information about predialysis nephrologist care and vascular access at the initiation of hemodialysis. On both forms, one of 82 causes is entered as the primary cause of ESRD, with identical options on the 1995 and 2005 versions. For this study, cases of ESRD due to ATN were those with the primary cause of ESRD listed as 'Tubular necrosis (no recovery)' on the Medical Evidence Report. The dates of death and first renal transplant were obtained from the Patients file, and the first listing for transplant was determined from the Waitlist_ki and Waitlist_ $\mathrm{kp}$ files. Renal recovery (a state in which dialysis and transplant were no longer necessary) was determined from the Rxhist file.

\section{Analysis}

US census data were used for population denominators for the years examined, with age in 5-year increments and race/ethnicity classified as non-Hispanic white, non-Hispanic black, Hispanic, and other [8]. The Poisson distribution was used to compute incidence rates of RRT-requiring ESRD due to ATN. For computation of standardized incidence ratios, expected incidence rates were estimated by applying the incidence rates in 2001-2002 for each discrete permutation of age, sex, and race-ethnicity to the corresponding subgroup of the US population in subsequent 2-year periods. Chi-square analysis was used for unadjusted comparisons of patients with and without ESRD due to ATN and logistic regression for adjusted comparisons. For comparisons of clinical outcomes of patients with and without ATN, patients were matched according to age in 1-year intervals, sex, race, and Hispanic ethnicity. Poisson regression was used to compute incidence rates and proportional hazards regression to compute adjusted hazards ratios (AHRs) for events occurring after the initiation of RRT, with follow-up ending on June 30, 2012. SAS, v9.2 (Cary, N.C., USA) being used for data analysis.

\section{Results}

In 2001-2002, 3,236 patients began maintenance RRT for ESRD attributed to ATN, a rate of 5.7 cases per million per year (PMPY; table 1); higher rates were seen with age 40-64 (6.1 PMPY) and $\geq 65$ (28.5 PMPY), male sex (6.8 PMPY), and non-Hispanic white (6.3 PMPY) and black race-ethnicity (7.1 PMPY). The proportion of ESRD attributed to ATN increased stepwise from 1.7 to $3.5 \%$ between 2001-2002 and 2009-2010. Standardized incidence ratios increased stepwise after 2001-2002, reaching 2.14 by $2009-2010$ in the overall population. Incidence trends were similar in all subgroups examined.

The characteristics of patients with ESRD attributed to ATN included older age ( $\geq 65$ years, 58.9 vs. $48.8 \%$ in patients without ATN), male sex (58.1 vs. $55.5 \%$ ), white race (81.6 vs. $65.1 \%$ ), and non-Hispanic ethnicity (93.1 vs. $86.7 \%)$. After adjusting for age, sex, race, and ethnicity, associations of ESRD attributed to ATN (vs. other causes; table 2) included more recent RRT initiation, older age, male sex, white race, non-Hispanic ethnicity, ischemic heart disease, lack of diabetes, alcohol abuse, drug abuse, hemodialysis for RRT, non-fistula access for hemodialysis, lack of nephrologist care, higher estimated glomerular filtration rate (eGFR), lower serum albumin levels, higher hemoglobin levels, and a longer interval between starting RRT and completion of a Medical Evidence Report.

Recovery of renal function (RRT was no longer required) occurred in $27.7 \%$ of patients with ATN, compared with $3.5 \%$ of patients without ATN; most recovery occurred in the first 3 months and recovery rates were higher than in matched controls without ATN (fig. 1; online suppl. table S1; for all online suppl. material, see www. karger.com/doi/10.1159/000369832). Among patients with ATN, with 2001-2002 as comparator (table 3), a stepwise increase in the likelihood of recovery occurred, especially after 2006, with unadjusted hazards ratios of 1.24 in 2007-2008 and 1.34 in 2009-2010. Recovery of renal function was also associated with a younger age, male sex, white race, lack of ischemic heart disease and diabetes, alcohol and drug abuse, hemodialysis for RRT, vascular access other than fistula for hemodialysis, shorter duration of nephrologist care, lower eGFR, body mass index, lower albumin and higher hemoglobin levels and earlier registration with the USRDS. Factors associated with hazards ratios above 2 for recovery included drug abuse (unadjusted hazards ratio [HR] 2.90), grafts (HR 3.82 vs. fistulas) and catheters (HR $4.99 \mathrm{vs}$. fistulas) for hemodialysis, and $\leq 12$ months of nephrologist care (HR 2.50). Hazards ratios were less than 0.5 for age $\geq 65$ years (HR 0.35 ), peri- 
Table 1. Standardized incidence ratios of ESRD attributed to acute tubular necrosis requiring renal replacement therapy, 2001-2010

\begin{tabular}{|c|c|c|c|c|c|}
\hline & 2001-2002 & $2003-2004$ & $2005-2006$ & 2007-2008 & $2009-2010$ \\
\hline US population, millions & 286 & 291 & 297 & 303 & 308 \\
\hline Cases of ESRD from ATN, $\mathrm{n}$ & 3,236 & 4,425 & 5,340 & 6,593 & 8,009 \\
\hline Proportion of ESRD due to ATN, \% & 1.7 & 2.2 & 2.5 & 3.0 & 3.5 \\
\hline Subgroup & Incidence rate & \multicolumn{4}{|c|}{ Standardized incidence ratio (vs. 2001-2002) } \\
\hline All & $5.7(0.1)$ & $1.32(0.02)$ & $1.55(0.02)$ & $1.84(0.02)$ & $2.14(0.02)$ \\
\hline \multicolumn{6}{|l|}{ Age, years } \\
\hline$<40$ & $0.7(0)$ & $1.32(0.08)^{\mathrm{a}}$ & $1.34(0.08)^{\mathrm{a}}$ & $2.00(0.09)$ & $2.19(0.10)$ \\
\hline $40-64$ & $6.1(0.2)$ & $1.49(0.04)$ & $1.73(0.04)$ & $2.11(0.04)$ & $2.43(0.04)$ \\
\hline$\geq 65$ & $28.5(0.6)$ & $1.29(0.03)$ & $1.52(0.03)$ & $1.74(0.03)$ & $2.06(0.03)$ \\
\hline \multicolumn{6}{|l|}{ Sex } \\
\hline Men & $6.8(0.2)$ & $1.26(0.03)$ & $1.52(0.03)$ & $1.79(0.03)$ & $2.05(0.03)$ \\
\hline Women & $4.5(0.1)$ & $1.42(0.03)$ & $1.59(0.03)$ & $1.91(0.04)$ & $2.28(0.04)$ \\
\hline \multicolumn{6}{|l|}{ Ethnicity/race } \\
\hline Non-Hispanic white & $6.3(0.1)$ & $1.32(0.02)$ & $1.57(0.02)$ & $1.88(0.03)$ & $2.20(0.03)$ \\
\hline Non-Hispanic black & $7.1(0.3)$ & $1.31(0.05)^{\mathrm{b}}$ & $1.51(0.05)$ & $1.70(0.05)$ & $1.97(0.06)$ \\
\hline Hispanic & $2.7(0.2)$ & $1.30(0.08)^{\mathrm{a}}$ & $1.36(0.07)^{\mathrm{b}}$ & $1.66(0.08)$ & $2.01(0.08)$ \\
\hline Other & $1.9(0.2)$ & $1.58(0.15)^{\mathrm{a}}$ & $1.54(0.14)^{\mathrm{a}}$ & $1.83(0.14)^{\mathrm{b}}$ & $2.09(0.15)$ \\
\hline
\end{tabular}

Parameter estimates are either rates per million per year or standardized incidence ratios (standardized to 2001-2002) with standard errors in parentheses. With PE denoting Point Estimate, CL Confidence Limit, Obs Observed Incidence Rate, Exp Expected Incidence Rate from rates seen in 2001-2002, standardized incidence ratios were estimated and reported as $\left(\mathrm{PE}_{\mathrm{Obs}} / \mathrm{PE}_{\mathrm{Exp}}\right)$ $\left(\left\{\left[95 \% \mathrm{CL}_{\mathrm{Obs}} / \mathrm{PE}_{\mathrm{Exp}}\right]-\left[5 \% \mathrm{CL}_{\mathrm{Obs}} / \mathrm{PE}_{\mathrm{Exp}}\right]\right\} / 3.92\right) \cdot \mathrm{p}$ values refer to comparisons of observed rates and those expected when those seen in 2001-2002 were applied to the years under consideration. $\mathrm{p}<0.001$ unless otherwise indicated. ${ }^{\mathrm{a}} 0.01 \leq \mathrm{p}<0.05$. ${ }^{\mathrm{b}} 0.001 \leq$ $\mathrm{p}<0.01$. ATN $=$ Acute tubular necrosis; ESRD = end-stage renal disease. toneal dialysis (HR $0.18 \mathrm{vs}$. hemodialysis) and $>8$ weeks to registration with the USRDS (HR 0.39 vs. $\leq 2$ ).

Over 2.4 years, $63.3 \%$ of patients with ATN died, and mortality rates were higher in this group than in the matched controls, overall and in most subgroups (fig. 1; online suppl. table S1). A stepwise decrease in the likelihood of death occurred, with unadjusted hazards ratios declining to 0.83 in 2009-2010 (table 3). Among patients with ATN (table 3), death was associated with older age, female sex, white race, non-Hispanic ethnicity, ischemic heart disease, diabetes, absence of alcohol and drug abuse, hemodialysis, catheters for hemodialysis, nephrologist care, higher eGFR, lower body mass index, lower albumin, lower hemoglobin and a longer interval between starting RRT and registration with the USRDS.

\section{Discussion}

We found that the incidence of treated ESRD attributed to ATN doubled over a 10-year period. Compared with patients with ESRD from other causes, white race

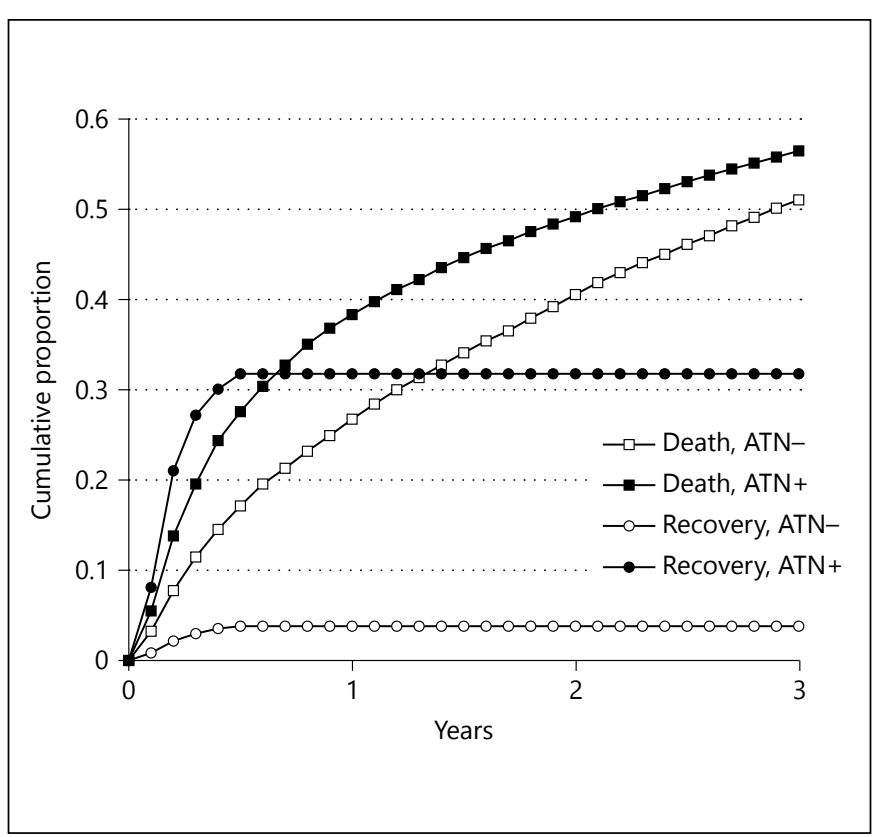

Fig. 1. The cumulative occurrence of renal recovery and death in patients with end-stage renal disease attributed to acute tubular necrosis (ATN) and matched controls without ATN. 
Table 2. Baseline characteristics at dialysis initiation

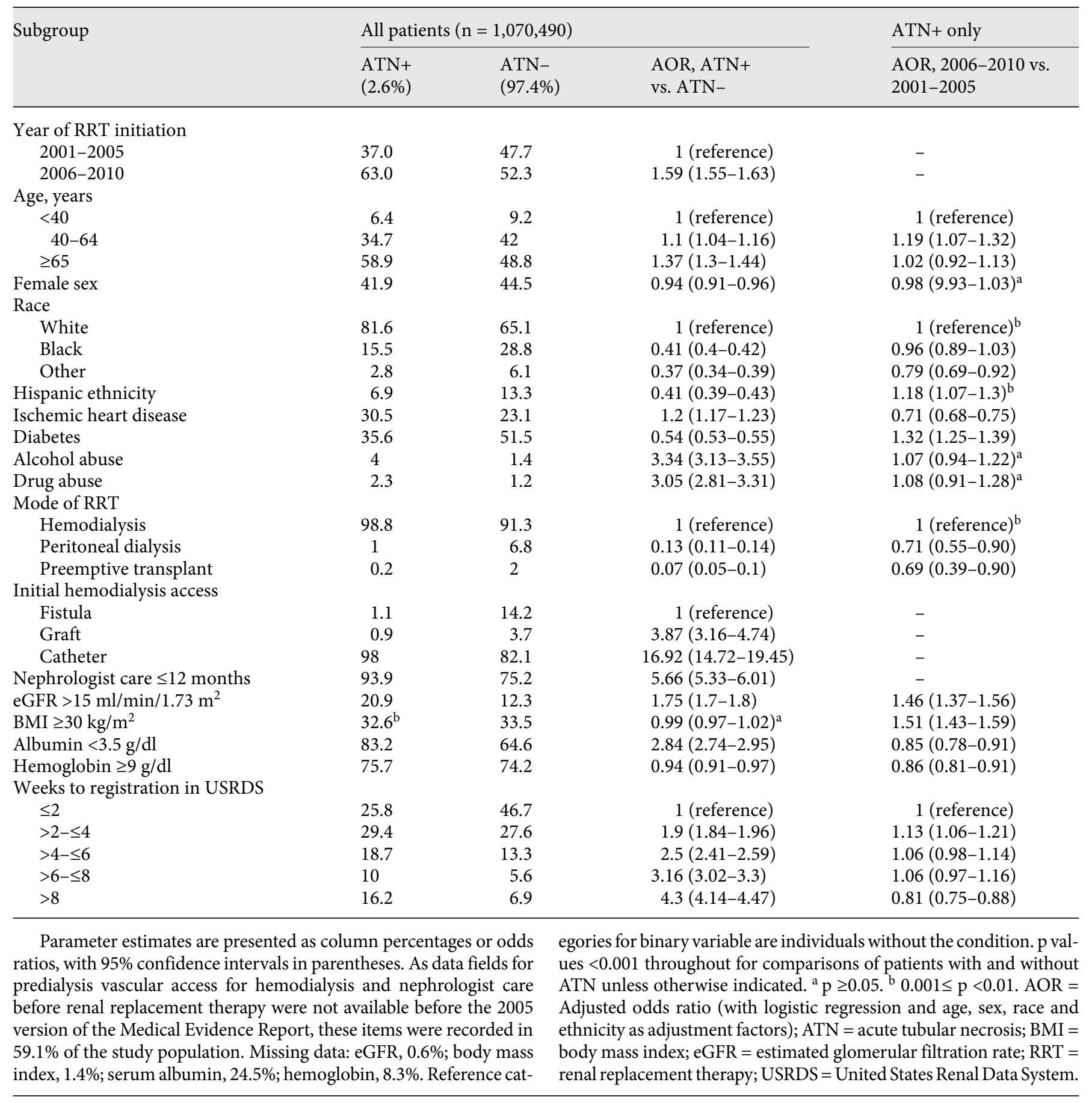

and non-Hispanic ethnicity were notably more likely. Over time, patients with ATN were more likely to initiate maintenance RRT at higher eGFR levels. Almost a third of patients with ATN regained independence from RRT, an occurrence that grew more likely in more recent years.
Despite the long recognition that failures of functional recovery can occur for all causes of AKI, few if any studies have examined the issue of ATN as a cause of ESRD. For example, a PubMed search on August 20, 2014, with the terms '(acute tubular necrosis) end stage (renal or kidney) 
Table 3. Hazards ratios for renal recovery and death, patients with end-stage renal disease attributed to acute tubular necrosis $(\mathrm{n}=27,603)$

\begin{tabular}{|c|c|c|c|c|c|}
\hline \multirow[t]{2}{*}{ Variable } & \multirow[t]{2}{*}{ Reference } & \multicolumn{2}{|c|}{ Renal recovery $(27.7 \%)$} & \multicolumn{2}{|l|}{ Death $(63.3 \%)$} \\
\hline & & unadjusted HR & adjusted HR & unadjusted HR & adjusted HR \\
\hline Year of RRT initiation & $2001-2002$ & & & & \\
\hline $2003-2004$ & & $1.02(0.93-1.12)^{\mathrm{a}}$ & $1.01(0.92-1.11)^{\mathrm{a}}$ & $0.98(0.93-1.03)^{\mathrm{a}}$ & $0.99(0.94-1.04)^{\mathrm{a}}$ \\
\hline $2005-2006$ & & $1.08(0.98-1.17)^{\mathrm{a}}$ & $1.07(0.98-1.17)^{\mathrm{a}}$ & $0.95(0.90-1.00)^{\mathrm{b}}$ & $0.95(0.90-0.99)^{\mathrm{b}}$ \\
\hline $2007-2008$ & & $1.24(1.14-1.35)$ & $1.22(1.12-1.32)$ & $0.87(0.83-0.92)$ & $0.89(0.84-0.93)$ \\
\hline 2009-2010 & & $1.34(1.24-1.45)$ & $1.32(1.22-1.44)$ & $0.83(0.79-0.87)$ & $0.84(0.80-0.88)$ \\
\hline Age, years & $<40$ & & & & \\
\hline $40-64$ & & $0.63(0.59-0.68)$ & $0.61(0.56-0.65)$ & $2.4(2.18-2.64)$ & $2.38(2.17-2.62)$ \\
\hline$\geq 65$ & & $0.35(0.33-0.38)$ & $0.33(0.3-0.35)$ & $4.73(4.31-5.18)$ & $4.67(4.26-5.13)$ \\
\hline Female sex & Male & $0.76(0.73-0.80)$ & $0.79(0.75-0.83)$ & $1.11(1.07-1.14)$ & $1.06(1.03-1.09)$ \\
\hline Race & White & & & & \\
\hline Black & & $0.81(0.76-0.87)$ & $0.69(0.65-0.74)$ & $0.86(0.83-0.90)$ & $1.01(0.97-1.05)^{\mathrm{a}}$ \\
\hline Other & & $0.81(0.70-0.94)^{\mathrm{c}}$ & $0.74(0.64-0.86)$ & $0.77(0.70-0.85)$ & $0.84(0.76-0.92)$ \\
\hline Hispanic ethnicity & Non-Hispanic & $0.93(0.85-1.02)^{\mathrm{a}}$ & $0.79(0.72-0.86)$ & $0.83(0.78-0.88)$ & $0.93(0.87-0.99)^{\mathrm{b}}$ \\
\hline Ischemic heart & Absent & $0.63(0.60-0.66)$ & $0.72(0.68-0.76)$ & $1.54(1.49-1.58)$ & $1.25(1.21-1.29)$ \\
\hline Diabetes & Absent & $0.73(0.69-0.76)$ & $0.78(0.74-0.82)$ & $1.24(1.20-1.28)$ & $1.16(1.12-1.19)$ \\
\hline Alcohol abuse & Absent & $1.86(1.69-2.04)$ & $1.41(1.28-1.55)$ & $0.74(0.68-0.81)$ & $1.04(0.95-1.13)^{\mathrm{a}}$ \\
\hline Drug abuse & Absent & $2.90(2.62-3.21)$ & $1.98(1.78-2.2)$ & $0.40(0.35-0.45)$ & $0.71(0.62-0.81)$ \\
\hline Peritoneal dialysis & Hemodialysis & $0.18(0.11-0.29)$ & $0.11(0.07-0.18)$ & $0.66(0.56-0.78)$ & $1.11(0.94-1.32)^{\mathrm{a}}$ \\
\hline $\mathrm{Graft}^{\mathrm{a}}$ & Fistula & $3.82(2.12-6.89)$ & $3.93(2.18-7.09)$ & $1.25(0.95-1.64)^{\mathrm{a}}$ & $1.38(1.05-1.82)^{\mathrm{b}}$ \\
\hline Catheter $^{\mathrm{a}}$ & Fistula & $4.99(3.00-8.28)$ & $5.11(3.08-8.48)$ & $1.29(1.06-1.58)^{\mathrm{b}}$ & $1.37(1.12-1.67)^{\mathrm{c}}$ \\
\hline Nephrologist care $\leq 12$ months $^{\mathrm{a}}$ & $\geq 12$ & $2.50(2.14-2.93)$ & $2.40(2.05-2.8)$ & $0.88(0.82-0.95)^{\mathrm{c}}$ & $0.97(0.89-1.04)^{\mathrm{a}}$ \\
\hline $\mathrm{eGFR}>15 \mathrm{ml} / \mathrm{min} / 1.73 \mathrm{~m}^{2}$ & $\leq 15$ & $0.89(0.84-0.94)$ & $0.83(0.78-0.88)$ & $1.17(1.13-1.21)$ & $1.25(1.20-1.29)$ \\
\hline $\mathrm{BMI} \geq 30 \mathrm{~kg} / \mathrm{m}^{2}$ & $<30$ & $1.27(1.21-1.33)$ & $1.24(1.18-1.3)$ & $0.87(0.84-0.90)$ & $0.91(0.89-0.95)$ \\
\hline Albumin $<3.5 \mathrm{~g} / \mathrm{dl}$ & $\geq 3.5$ & $1.12(1.04-1.20)^{\mathrm{c}}$ & $1.16(1.08-1.24)$ & $1.19(1.14-1.25)$ & $1.16(1.11-1.22)$ \\
\hline Hemoglobin $\geq 9 \mathrm{~g} / \mathrm{dl}$ & $<9.0$ & $1.23(1.16-1.30)$ & $1.26(1.19-1.33)$ & $0.94(0.90-0.97)$ & $0.91(0.87-0.94)$ \\
\hline Weeks to registration in USRDS & $\leq 2$ & & & & \\
\hline$>2-\leq 4$ & & $1.19(1.12-1.26)$ & $1.21(1.14-1.28)$ & $0.99(0.95-1.03)^{\mathrm{a}}$ & $0.98(0.94-1.02)^{\mathrm{a}}$ \\
\hline$>4-\leq 6$ & & $0.94(0.88-1)^{\mathrm{a}}$ & $0.95(0.88-1.01)^{\mathrm{a}}$ & $1.06(1.01-1.11)^{\mathrm{b}}$ & $1.05(1-1.09)^{\mathrm{a}}$ \\
\hline$>6-\leq 8$ & & $0.76(0.7-0.83)$ & $0.77(0.71-0.84)$ & $1.08(1.02-1.14)^{c}$ & $1.05(1-1.11)^{\mathrm{a}}$ \\
\hline$>8$ & & $0.39(0.36-0.43)$ & $0.38(0.35-0.42)$ & $1.07(1.02-1.12)^{\mathrm{c}}$ & $1.08(1.03-1.13)^{\mathrm{C}}$ \\
\hline
\end{tabular}

$\mathrm{p}$ values $<0.001$ throughout unless otherwise indicated. ${ }^{\mathrm{a}} \mathrm{p} \geq 0.05 .{ }^{\mathrm{b}} 0.01 \leq \mathrm{p}<0.05$. $^{\mathrm{c}} 0.001 \leq \mathrm{p}<0.01$. BMI $=$ Body mass index; eGFR $=$ estimated glomerular filtration rate; $\mathrm{HR}=$ hazards ratio (with Cox regression and age, sex, race, and ethnicity as adjustment factors and 95\% confidence intervals in parentheses); RRT = (year of initiation of) renal replacement therapy; USRDS = United States Renal Data System.

dialysis' failed to generate a single relevant citation. Similarly, examination of other national ESRD registries proved to be non-informative. Between 1984 and 1995, a single-center study from the United Kingdom examined patients with severe AKI, of whom $16.2 \%$ remained dependent on long-term dialysis. The likelihood of non-recovery of renal function varied by underlying cause of $\mathrm{AKI}$, and as in this study, survival on dialysis was inferior to survival with other causes of ESRD [9].

Delivery of longer-term outpatient dialysis to patients with AKI has become a more pressing issue over the past 10 years. A particularly difficult problem can arise when hospitals and patients seek discharge, and provision of dialysis is the only reason for continued hospitalization. The interplay between AKI, maintenance dialysis, private insurance, Medicare, and the USRDS is multifaceted. Regardless of future intentions to use Medicare for the disbursement of dialysis expenses, a Medical Evidence Report (form CMS-2728) must be filed within 45 days of the first maintenance dialysis session [10]. Also, new Medicare coverage for in-center patients becomes available after 3 months of in-center hemodialysis. In the Medicare setting, for example, only facilities with expertise in dialysis treatment therapy are reimbursed. Thus, some hospitals arranged for dialysis to be provided in outpatient facilities, billed Medicare, and paid the facilities under con- 
tractual arrangements. In July 2012, however, CMS made it clear that 'ESRD facilities cannot furnish acute dialysis to hospital outpatients' [11]. While our study cannot determine the relative contributions of biological causation and administrative strategy to our main findings, a 2 -anda-half-fold increase in incidence rates is impressive; functionally, this study suggests that a substantial proportion of the patients do not have irreversible kidney disease and that the increased incidence reflects factors other than true ATN.

Our study has several limitations. This was a non-experimental study of associations, and causes and effects cannot be separated with confidence. It is retrospective and registry-based and lacks desirable data elements that a well-designed prospective study could provide. In particular, even when a given comorbid illness was identified as being present, the severity of this illness was not evaluated. All things being equal, it is plausible that the severity of known comorbid illnesses and the likelihood of unknown comorbid illnesses were greater with ATN, potential explanatory hypotheses for the poor outcomes in comparison to other causes of ESRD. A true tissue diagnosis and accurate information regarding renal size, GFR, and urine output would be required to refute the hypothesis that the study findings are driven by non-biological factors.

Despite its limitations, this study may provide some worthwhile information. The intersection of AKI, ESRD, and long-term outpatient dialysis provision appears to be an emerging and unmet health care need. Our sample size was large, a useful feature when attempting to identify population trends and major risk associations. If our findings are valid, they may help inform decision making and, possibly, the configuration of ESRD services.

\section{Acknowledgments}

This study was performed as a deliverable under Contract No. HHSN267200715002C (National Institute of Diabetes and Digestive and Kidney Diseases, National Institutes of Health, Bethesda, Md., USA). The data reported here have been supplied by the USRDS. The interpretation and reporting of these data are the responsibility of the authors and in no way should be seen as an official policy or interpretation of the US government. The authors thank Chronic Disease Research Group colleagues Beth Forrest for regulatory assistance, Delaney Berrini, BS, for manuscript preparation, and Nan Booth, MSW, MPH, and ELS for manuscript editing.

\section{References}

$>1$ Thakar CV, Christianson A, Freyberg R, Almenoff $\mathrm{P}$, Render ML: Incidence and outcomes of acute kidney injury in intensive care units: a Veterans Administration study. Crit Care Med 2009;37:2552-2558.

-2 Chertow GM, Burdick E, Honour M, Bonventre JV, Bates DW: Acute kidney injury, mortality, length of stay, and costs in hospitalized patients. J Am Soc Nephrol 2005;16:33653370 .

-3 Ishani A, Nelson D, Clothier B, Schult T, Nugent S, Greer N, Slinin Y, Ensrud KE: The magnitude of acute serum creatinine increase after cardiac surgery and the risk of chronic kidney disease, progression of kidney disease, and death. Arch Intern Med 2011;171:226233.
4 Ishani A, Xue JL, Himmelfarb J, Eggers PW, Kimmel PL, Molitoris BA, Collins AJ: Acute kidney injury increases risk of ESRD among elderly. J Am Soc Nephrol 2009;20:223-228.

$\checkmark 5$ Wald R, Quinn RR, Adhikari NK, Burns KE, Friedrich JO, Garg AX, Harel Z, Hladunewich MA, Luo J, Mamdani M, Perl J, Ray JG: Risk of chronic dialysis and death following acute kidney injury. Am J Med 2012;125:585-593.

$\checkmark 6$ Coca SG, Singanamala S, Parikh CR: Chronic kidney disease after acute kidney injury: a systematic review and meta-analysis. Kidney Int 2012;81:442-448.

7 Liangos O, Wald R, O’Bell JW, Price L, Pereira BJ, Jaber BL: Epidemiology and outcomes of acute renal failure in hospitalized patients: a national survey. Clin J Am Soc Nephrol 2006; $1: 43-51$.

8 US Census Bureau: State Intercensal Estimates (2000-2010), 2013. Available at http://www. census.gov/popest/data/intercensal/state/ state2010.html (accessed October 28, 2014).
9 Bhandari S, Turney JH: Survivors of acute renal failure who do not recover renal function. QJM 1996;89:415-421.

10 US Department of Health and Human Services: End Stage Renal Disease Medical Evidence Report Medicare Entitlement and/or Patient Registration: Form CMS-2728-U3; in Centers for Medicare and Medicaid Services, 2006. Available at http://www.cms.gov/Medi care/CMS-Forms/CMS-Forms/downloads/ CMS2728.pdf (accessed October 28, 2014).

11 Can Certified ESRD Facilities Furnish Acute Dialysis to Hospital Outpatients? Available at https://www.cms.gov/Medicare/MedicareFee-for-Service-Payment/HospitalOut patientPPS/Downloads/Acute-Dialysis-Site. pdf (accessed October 28, 2014). 